\title{
Digital è public? L'esempio di alcune banche dati a contenuto storico
}

\author{
di Igor Pizzirusso
}

Apr 16, $2020 \mid \underline{\text { In evidenza, }}$ Storia pubblica $|\underline{0}|$

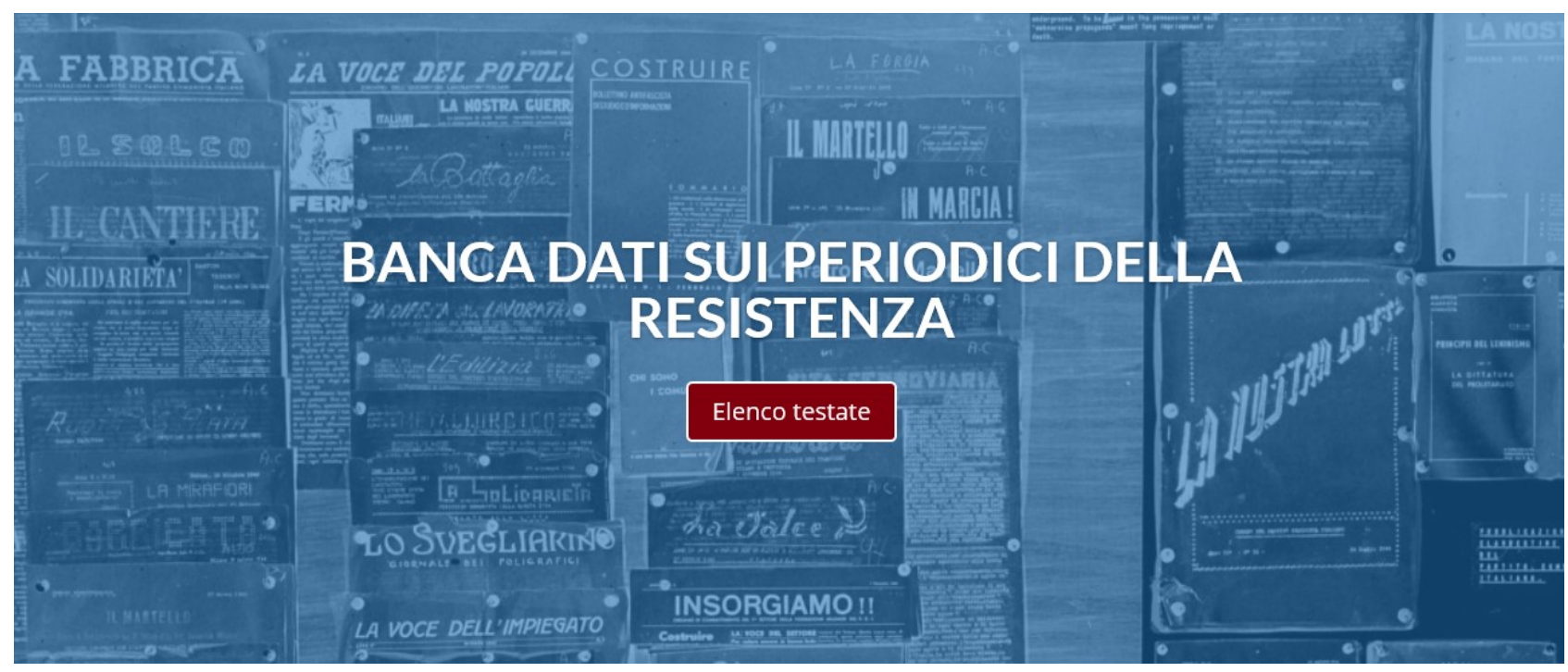

Dettaglio dell'home page del database www.stampaclandestina.it

\begin{abstract}
L'articolo riflette sulla differenza tra i concetti di digital history e di digital public history, molto chiara nel dibattito internazionale in materia, ma ancora oggetto di molteplici fraintendimenti nello scenario italiano. L'autore sviluppa i concetti espressi da Thomas Cauvin nel 2016 (Public History. A textbook of practice), applicandoli ad alcuni esempi pratici: una banca dati sulle fonti (www.stampaclandestina.it), una serie di banche dati sulle biografie (www.ultimelettere.it e www.antifascistispagna.it) e due banche dati nate da presupposti fortemente diversi, ma entrambe con una forte impronta memorialistica e territoriale (http://www.straginazifasciste.it e www.pietrenellarete.it).
\end{abstract}

\section{Introduzione}

Una citazione attribuita al noto esponente della confessione evangelica Billy Sunday (oltre che allo psicologo canadese Peter J. Laurence) recita pressappoco così: «Andare in chiesa non fa di nessuno un Cristiano, più di quanto andare in garage non faccia di lui un'automobile»[1]. Qualcosa di simile vale per il web: pur non essendo né un luogo di culto né un parcheggio, esso rappresenta uno spazio (benché virtuale e non fisico) nel quale non conta semplicemente che alcuni oggetti vi stazionino e vi si muovano, ma come lo facciano e in che modo si approccino al pubblico.

In quest'ottica le banche dati sono forse lo strumento più complesso e interessante da esaminare. Nate come utili sistemi di archiviazione e catalogazione, si sono evolute prima in formidabili mezzi di conservazione dei patrimoni e di supporti alla ricerca e poi in prodotti digitali assolutamente complessi, in grado di aggiungere gli aspetti più moderni della comunicazione storica[2] alle loro caratteristiche originarie di sistemi prettamente archivistici e catalogativi. 
Ciò nonostante si può dire siano tutte catalogabili come strumenti di public history? $\mathrm{O}$ invece è opportuno distinguere quali lo siano, quali potrebbero esserlo e quali invece non ne abbiano assolutamente le caratteristiche?

\section{Digital humanities e Digital history}

Prima di procedere con l'analisi però, è bene delineare con maggiore chiarezza definizioni, insiemi e sottoinsiemi. La digital history appartiene al novero delle Digital humanities, ma non ne è un sinonimo. Nelle Digital humanities sono, infatti, comprese pratiche di analisi dei testi mediante strumenti informatici attive già dagli anni Cinquanta e Sessanta del Novecento. [3] All'epoca si parlava di humanities computing, mentre il termine odierno si è affermato a partire dal 2004 ed ha un'accezione più ampia che include le rappresentazioni visuali (infografiche et similia) e dinamiche di partecipazione pubblica.[4]

La digital history attiene a qualcosa di più circoscritto, che presta meno attenzione agli aspetti linguistici e più a quelli storici e contenutistici. Nata inizialmente e principalmente per indicare un insieme di strumenti per la raccolta, la catalogazione e la restituzione delle fonti online, a supporto e ad agevolazione della ricerca storica classica, con il tempo essa si è evoluta insieme al resto del web, abbracciando in pieno la comunicazione storica a tutto tondo.[5] Non a caso, nel 2008, alcuni operatori del settore avevano definito la digital history «tutto ciò (metodi di ricerca, articoli di riviste, monografie, blog, esercizi in classe) che usa le tecnologie digitali per creare, accrescere o distribuire ricerche e sapere storico».[6]

Una definizione onnicomprensiva, insomma, che però aiuta a rendere l'idea di come ormai la digital history non vada più ritenuta come un mero insieme di strumenti e pratiche, o di semplici supporti alla ricerca, ma un nuovo modo di produrre contenuti storici[7], con nuovi soggetti protagonisti, nuovi pubblici potenziali e nuovi risultati.

\section{Pubblici reali, pubblici potenziali}

È notorio che ogni contenuto o informazione veicolata sul web in modo gratuito e senza censura abbia la possibilità di essere vista e letta da chiunque. Il pubblico di riferimento è quindi vastissimo e il confine è rappresentato solo dal mondo connesso alla rete. E tuttavia questa universalità è più una mera opportunità che un dato reale; essere sul web non ha un'immediata traduzione in una reale platea di ascoltatori o lettori o visitatori. Un conto è infatti il "pubblico accesso" (dal termine anglosassone public access[8], contempla l'eventualità che chiunque possa seguire o leggere un prodotto digitale), un altro sono l'accesso e la consultazione vere e proprie. Ad esempio esistono ricerche storiche valide e innovative che ciò nondimeno rimangono confinate a un ristretto gruppo di interlocutori[9], o perché la loro natura complessa le rende poco fruibili e spendibili presso il cosiddetto "grande pubblico", o per il modo in cui vengono restituite, presentate o narrate.

Occorre, dunque, ragionare proprio sulle pratiche di trasmissione dei contenuti e delle informazioni e su quanto un prodotto storico digitale riesca a creare seguito, ascolto, lettura, o persino a spingere il pubblico a partecipare attivamente alla sua costruzione o al suo perfezionamento. In altre parole occorre comprendere quanto il prodotto storico veicolato nel web sia capace di coinvolgere i suoi fruitori, di impegnarli o di "ingaggiarli" (per usare un anglicismo che deriva dal verbo inglese to engage e al quale si lega non a caso l'espressione anglosassone public engagement [10]). 
In pratica, e se intendiamo la public history in senso forte - e non come spesso accade con l'accezione che rimanda a una buona comunicazione o divulgazione storica - è soltanto attraverso il public engagement che la digital history si trasforma/si evolve in public history.[11]

\section{Il web partecipato}

Le tecnologie del cosiddetto web 2.0 hanno facilitato l'instaurarsi del public engagement. Social network, piattaforme di blogging e ambienti Wiki (di cui Wikipedia è solo l'esempio più noto ed eminente) incentivano per loro stessa natura un meccanismo di partecipazione attiva del pubblico[12]. Anche in questo caso tuttavia l'opzione tecnologica non è condizione esaustiva per ottenere coinvolgimento. Per quanto, riprendendo le parole di David Bidussa[13], oggi vi sia grande richiesta di sapere storico presso il pubblico, fruire di contenuti storici - che siano digitali o di altro tipo - presuppone un livello di cultura e consapevolezza sempre meno comuni. Ne deriva che argomentazioni molto tecniche o ambiti scarsamente noti e appetibili possono avere l'effetto di scoraggiare l'interesse anche del più fanatico degli appassionati.

Così come in altri ambiti pure nel web modalità e pratiche con cui vengono elaborati e presentati i contenuti storici risultano fondamentali per interessare e coinvolgere il pubblico. È perciò inevitabile che un prodotto digitale basato sulla pubblicazione di fonti abbia meno appetibilità di un altro incentrato sui percorsi biografici o sulle memorie collettive.

Il discorso vale, per altro, anche se lo applichiamo al lavoro dei docenti in classe e pensiamo agli studenti come a un pubblico che si ha intenzione di coinvolgere. È sicuramente più facile interessarli quando costruiamo percorsi o laboratori didattici che sfruttano la potenza narrativa delle biografie o la pervasività delle memorie (individuali o della comunità in cui la scuola è inserita). È per questo motivo che gli esempi riportati, pur mirando a riflettere in prima battuta sulla digital public history, costituiscono un'utile riflessione anche per gli insegnanti che si pongano domande sull'uso di strumenti come questi nel lavoro in classe.

\section{Storia e memoria secondo il public historian}

Introdurre elementi legati alla memoria ha sempre l'effetto, come meglio vedremo in seguito, di facilitare l'interazione con il pubblico e il suo coinvolgimento. Serge Noiret, attuale Presidente dell'Associazione italiana di Public History (ed ex Presidente della Federazione internazionale), qualche anno fa scriveva:

Il Public Historian ripudia la distinzione fatta dagli storici accademici tra "storiografia e memoria" (sia individuali che collettive) perché ritiene che entrambe siano manifestazioni del passato da custodire, analizzare e promuovere nella loro complessità: la memoria non è una forma di cecità e di impermeabilità alla storia "vera", unica versione colta e scientifica della lettura del passato che viene identificata con la storiografia prodotta alla luce delle fonti tradizionali.[14]

Se però digital history e public history non sono la stessa cosa, nemmeno lo sono public history e public memory. Per quanto infatti storia e memoria possano procedere insieme, esse non sono né saranno mai un'unica entità. La loro sovrapposizione, che è una tendenza sempre più frequente oggi giorno, rischia di diventare pericolosa, come lo stesso Noiret ha rilevato più di recente[15]. Il ruolo della public history (e degli strumenti a essa afferenti) è allora forse quello di costruire un ponte tra 
le due, contribuendo a ripulire la memoria dalle contaminazioni e a contestualizzarla in un quadro di riferimento complessivo storicamente più accurato.

Ma questa è un'altra storia...[16]

\section{Le banche dati dell'Istituto nazionale Ferruccio Parri}

Nel corso degli ultimi anni, l'Istituto nazionale Ferruccio Parri e gli enti associati alla sua rete hanno prodotto diversi strumenti digitali, nati da progetti di catalogazione delle fonti, di divulgazione, di raccolta documentale o biografica, ricerca storica propriamente detta. I livelli sono perciò molteplici e ogni prodotto presenta le sue specificità e i suoi tratti distintivi, che sono per altro utili ad analizzare i concetti eunciati finora.

Nel dettaglio, verranno presi in esame una banca dati sulle fonti (www.stampaclandestina.it), due banche dati biografiche (www.ultimelettere.it e www.antifascistispagna.it) e due banche dati nate da presupposti fortemente diversi, ma entrambe con una forte impronta memorialistica e territoriale (www.straginazifasciste.it e www.pietrenellarete.it, quest'ultima a cura dell'Associazione PopHistory).

\section{Banca dati con le fonti: stampa clandestina}

La banca dati Stampa clandestina 1943-1945 (www.stampaclandestina.it) raccoglie e indicizza i periodici prodotti sotto l'occupazione tedesca e la dittatura fascista, in condizioni di illegalità e spesso con mezzi di fortuna. L'importanza storica e culturale di questo materiale fu evidente già nell'immediato dopoguerra (si pensi alla circolare inviata con grande attenzione e lungimiranza dal CLN nel novembre del 1945, con gli echi della guerra e dell'occupazione ancora nelle orecchie). 


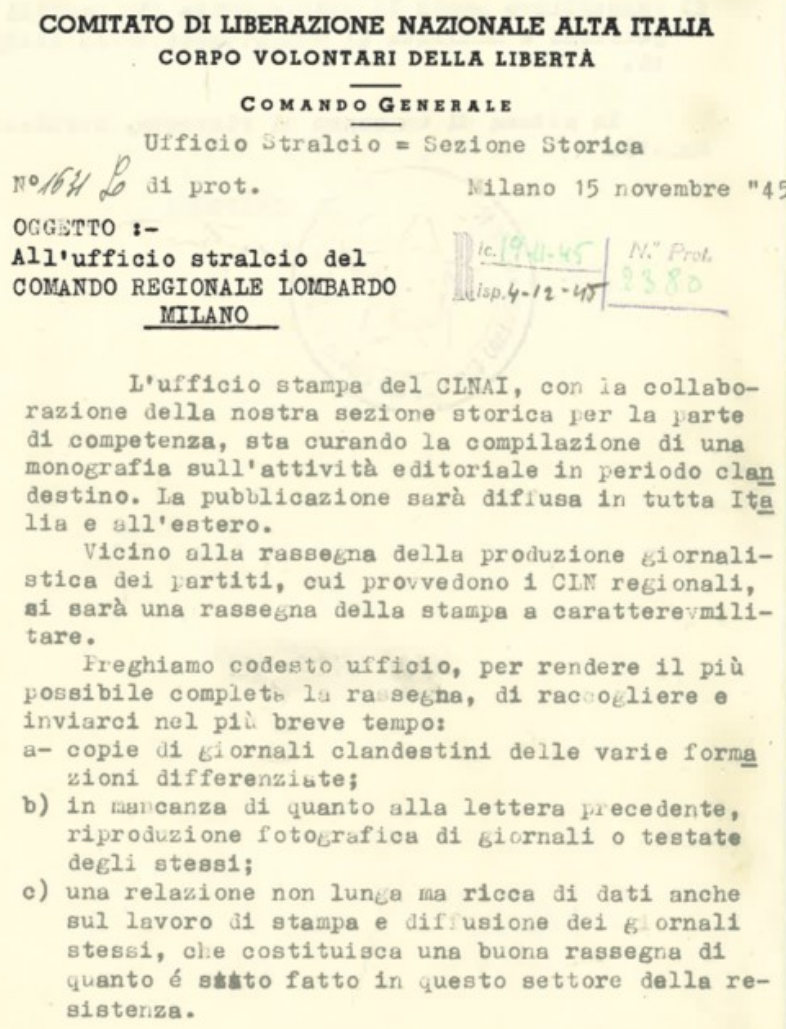

COMITATO DI LIBERAZIONE NAZIONALE ALTA ITALIA CORPO VOLONTARI DELLA LIBERTA COMANDO GENEARLE

Lettera inviata dall'Ufficio stralcio del Clnai, datata 15 novembre 1945, relativa al censimento e alla raccolta della stampa clandestina.

Il documento è conservato nell'Archivio dell' Istituto nazionale Ferruccio Parri, Fondo CVL, b. 45, fasc 99

D'altro canto va ricordato quanto fosse importante rivendicare la libertà di parola in un paese che non solo aveva subito soltanto la difficile oppressione nazifascista, ma che aveva vissuto i precedenti venti anni sotto il giogo della dittatura fascista. Lo scopo della stampa clandestina è esplicito proprio in quest'ottica e dichiarato con forza da Carlo e Nello Rosselli nell'articolo introduttivo al primo numero di «Non Mollare», stampato in clandestinità a Firenze nel gennaio del 1925: «Non ci è concessa la libertà di parola: ce la prendiamo».

\section{Una chiamata pubblica}

La stampa clandestina rappresenta, dunque, un inestimabile patrimonio storico, culturale e linguistico (su queste testate scrivono alcune delle penne più brillanti dell'epoca), che tuttavia vive per sua stessa natura in condizioni di estrema precarietà: le tirature sono limitate per la scarsità di mezzi con cui vengono stampati i giornali e spesso gli esemplari vanno incontro a distruzione perché essere scoperti in possesso di questo materiale da parte dei nazifascisti portava alla cattura e, in qualche caso, alla morte.[17] 
La circolare del CLN del 1945 assume quindi a tutti gli effetti i connotati di una chiamata pubblica. E tuttavia l'intenzione non ha il successo sperato. Basti considerare l'estrema parzialità e la frammentarietà delle collezioni note fin qui e pubblicate nel corso degli anni in cataloghi nazionali o ricerche (e banche dati) su base locale o riferite a specifici corpora archivistici conservati presso un ente o un'istituzione culturale.

Lo scopo della banca dati Stampa clandestina è proprio quello di superare queste parzialità e raccogliere in un unico luogo tutte le collezioni di testate attualmente note, edite e non, per dare pubblico accesso a fonti preziose per la ricerca attraverso un prodotto di digital history.

\section{Uno strumento per specialisti}

Nonostante l'indubbio valore storico e documentale del progetto, la stampa clandestina (e la relativa banca dati) resta un materiale indagato prevalentemente da una platea di storici e specialisti. Il public engagement resta difficile, perché la maggior parte delle persone si sente poco attirata da una documentazione poco divulgata, poco nota e la cui importanza è, forse, scarsamente percepita. Per gli stessi motivi il pubblico potenziale si sente poco coinvolto a partecipare alla costruzione del progetto, dal momento che le copie dei periodici clandestini rimaste negli archivi privati sono pochissime.

Una situazione paradossale, se pensiamo che la stampa clandestina nasce e si sviluppa per essere diffusa e letta, quindi per raggiungere un pubblico più vasto possibile con la grande forza comunicativa, tipica della propaganda.

Il problema si riflette anche nella spendibilità dello strumento informatico a scuola, da parte dei docenti. Nel database è inserito un dossier didattico, che tuttavia è poco valorizzato difficilmente raggiungibile. Al suo interno sono elencati alcuni possibili percorsi di indagine e utilizzo delle testate catalogate nella banca dati, che però vengono delineati in termini piuttosto vaghi e con pochissime indicazioni di carattere pratico. Attuarli a scuola richiede quindi conoscenze specifiche sul tema nonché tempo per analizzare il corpus documentario. Un lavoro lungo, pur con l'agevolazione di tutti i filtri di ricerca del database che consentono di categorizzare (e pertanto distinguere) le testate in base a diversi criteri.

\section{Banche dati biografiche: "Ultime lettere" e "Data Spanish Civil War"}


ultume lettere"

di condannati a morte e di

deportatu della Resistenza italiana
La base di dati Ultime lettere di condannati a morte e di deportati della Resistenza italiana, pubblicata online il 26 aprile 2007, si propone di raccogliere il più vasto - e per quanto possibile esaustivo - archivio virtuale di documenti di tal genere. Essa è costituita principalmente dal materiale contenuto nei fondi archivistici donati all'stituto Nazionale per il Movimento di Liberazione in Italia da Piero Malvezzi (tra it 1985 e il 1986) e da Mimmo Franzinelli (nel 2005), ma nel corso degli anni si è arricchita di numerosa altra documentazione, messa a disposizione da archivi di enti e associazioni e da privati (nella maggior parte dei casi famigliari delle vittime).

L'archivio "virtuale" (che si è ampliato col tempo fino a includere anche i "Testamenti spirituali") è tuttora in corso di alimentazione. Per contribuire alla ricerca in corso, segnalando l'esistenza di documenti $e / 0$ fotografie, dati imprecisi o integrativi nelle schede personali dei condannati, scrivete alla redazione compilando il modulo dei contatti.

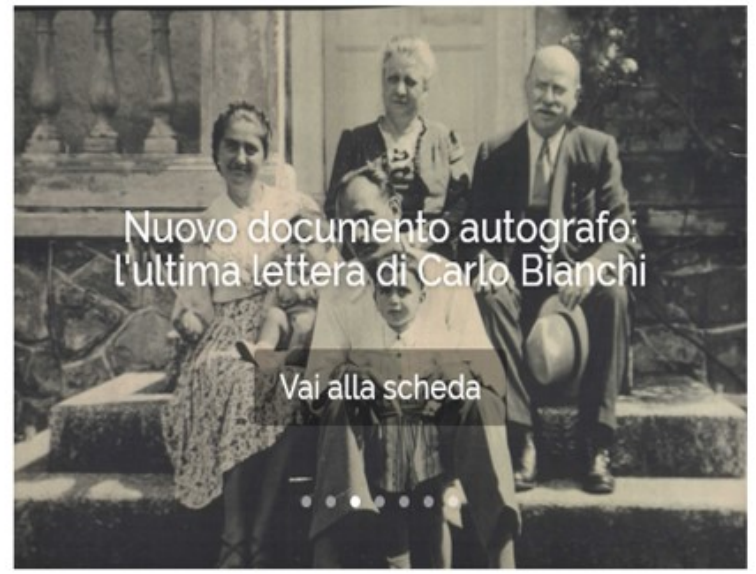

Consulta la banca dati

La banca dati www.ultimelettere.it raccoglie i documenti scritti da partigiani, civili legati alla Resistenza (parenti o fiancheggiatori) e deportati politici e razziali nell'imminenza della morte. Si tratta, dunque, di un database che abbina l'elemento biografico a quello documentale e che, però, non ha le caratteristiche di una vera e propria "fonte di ricerca", se non con le dovute e necessarie mediazioni. Nel delicato momento dell'imminenza della morte i racconti dei protagonisti sono influenzati dalla soggettività e vanno collocati nel contesto in cui sono stati scritti, con i necessari raffronti fattuali e l'ausilio di altre fonti di ricerca scritte, le quali servono «soprattutto a disporre di un quadro problematico ma plausibile degli eventi, su cui verificare e misurare il lavoro creativo della memoria e del racconto»[18].

Nella presentazione al progetto Gianni Perona - direttore dei lavori e all'epoca Direttore scientifico dell'Istituto nazionale Ferruccio Parri - dichiara espressamente il valore di questi documenti:

Le lettere di un condannato a morte, in un contesto di persecuzione a carattere politico o religioso, hanno assunto ripetutamente nella storia europea un carattere di documento insieme privato e pubblico, di rivendicazione d'innocenza e di testimonianza, capace di trasmettere ai posteri un 'immagine onorevole per la famiglia ed esemplare per i compagni di fede.

Il raccogliere tali lettere approdò spesso - ad esempio presso i protestanti nelle guerre di religione - alla costituzione di un vero e proprio martirologio, e certamente a questo obiettivo mirarono anche quei militanti dei movimenti di resistenza che un po' dappertutto nell'Europa appena liberata, talora a guerra non ancora finita, ricercarono gli estremi messaggi dei loro compagni, e li riunirono e pubblicarono.[19]

Dal volume Lettere di condannati a morte della Resistenza italiana nasce lo stimolo al lavoro: restituire in forma digitale il corpus documentario raccolto nel ventennio fra gli anni Cinquanta e gli anni Settanta da Giovanni Pirelli e Piero Malvezzi. Un patrimonio che sarà poi donato da Malvezzi all'Istituto nazionale Ferruccio Parri che conserva copia di tutti i documenti editi nel volume cartaceo. 


\section{Una ricerca d'altri tempi}

All'interno del fondo archivistico, tuttavia, gli originali sono rari. La maggior parte delle lettere sono conservate come trascrizioni a macchina, da collaboratori dei curatori. Dietro a questa scelta, ci sono innanzitutto questioni tecniche: le trascrizioni erano l'unica possibilità per procurarsi i testi. Malvezzi e Pirelli, ben consci del valore che avevano tali documenti per i famigliari (mogli e mariti, figli e figlie, padri e madri) non chiedevano gli originali sapendo che una simile richiesta avrebbe inibito i possessori a divulgare la documentazione. C'erano poi anche questioni storiche e sociali. Negli anni Cinquanta il principale intento nel diffondere questi scritti aveva matrice idealizzante[20] e la trascrizione aveva il vantaggio di potere operare censure sia sulle parti ritenute più «banali» (legate alla quotidianità della prigionia) sia su quelle più compromettenti (come richieste di vendetta o ripensamenti).

Non è un caso che il lavoro di Malvezzi e Pirelli si concentri soprattutto sulla ricerca del testo delle lettere, mentre poca attenzione sia dedicata non solo al documento originale, ma anche alla biografia dello scrivente al di fuori del momento del suo sacrificio. C'era dunque un intento chiaramente «pubblico», utile a generare empatia e coinvolgimento e ad alimentare processi di matrice celebrativa e memoriale. Ma un po' distante dagli intenti della ricerca storica.

\section{La ricerca di questi tempi}

Ultime lettere cerca di ridefinire i criteri dell'opera di riferimento in cartaceo redigendo schede biografiche più puntuali e precise, con un' accurata ricostruzione e un inquadramento delle vicende nel quadro storico. L'ampio uso del digitale nel reperimento dei documenti, unito al maggior distacco temporale ed emotivo dei possessori odierni, hanno dato vita a una ricerca differente rispetto a quella di Malvezzi e Pirelli. Una ricerca che considera la lettera un vero e proprio reperto storico, da restituire nella sua integralità e utile a fornire fondamentali dati di contorno, come ad esempio il supporto spesso non convenzionale su cui i messaggi sono stati scritti (come cambia la grafia, quali errori, quali omissioni o correzioni, ecc.). 

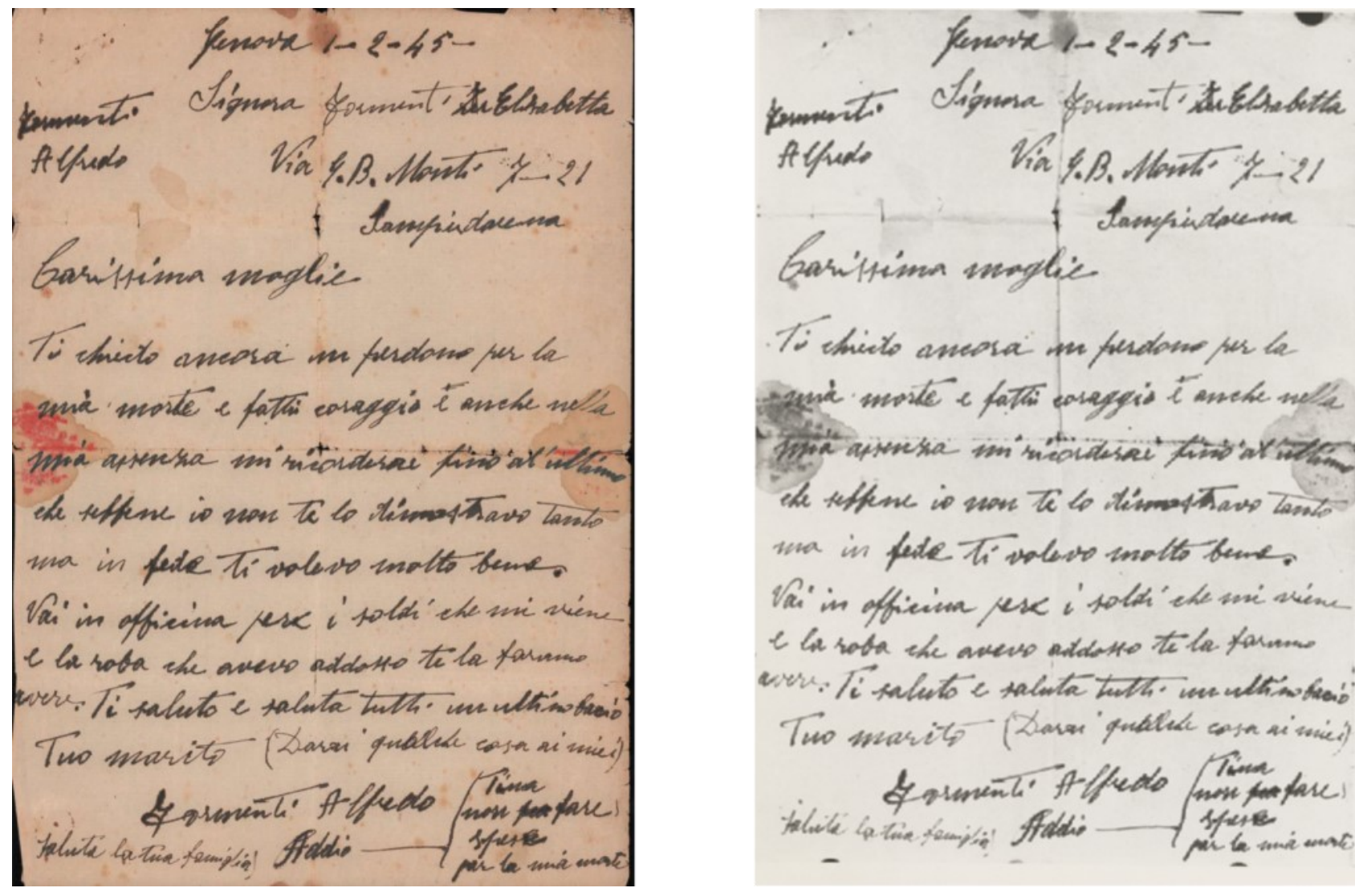

A sinistra l'originale autografo dell'ultima lettera di Alfredo Formenti; sono visibili le macchie di sangue che nella fotografia in bianco e nero (a destra) sembravano inchiostro.

La banca dati www.ultimelettere.it è oggi uno strumento di digital history di enorme diffusione. Nel corso degli anni diversi attori sono intervenuti indicando dati da correggere o offrendo originali autografi che non erano ancora stati reperiti. In questo senso il digitale è stato fondamentale per la fluidità e per la possibilità di interventi continui che ha consentito e ancora consente. E tuttavia la grande partecipazione e il seguito suscitati dal tema esulano dallo strumento. Il volume di Malvezzi e Pirelli conta ben 14 edizioni dalla prima del 1952: il pubblico è quindi "ingaggiato" indipendentemente dallo strumento, anche se si può dire che la forma virtuale funge senz'altro altro da cassa di risonanza.

\section{Oggi in Spagna, domani in Italia}

Data Spanish Civil War (www.antifascistispagna.it) raccoglie le biografie degli antifascisti, volontari e combattenti della guerra di Spagna: un evento centrale nella storia del primo Novecento, benché spesso sottovalutato nella sua importanza. Nel conflitto combattuto dal 1936 al 1939 si polarizzano gli schieramenti che saranno poi quelli della Seconda guerra mondiale ed emerge la debolezza britannica e francese che concorrerà a precipitare il continente nel conflitto. Ma la Spagna è anche il luogo dove, per gli Italiani, viene trasferita ed esplode la conflittualità tra totalitarismo e oppositori e che da qui promette di dilagare in Europa. "Oggi in Spagna, domani in Italia» è la frase pronunciata da Carlo Rosselli a Radio Barcellona, nel novembre del 1936. Un auspicio che dovrà tuttavia attendere il 1943 e la Resistenza per avverarsi. 
Per quanto il dato numerico degli antifascisti combattenti italiani in Spagna che furono poi protagonisti anche della lotta di liberazione in Italia si attesti statisticamente solo intorno al 10 per cento (meno di quattrocento su oltre quattromila)[21], è indubbio che vi siano diversi elementi di continuità, quanto meno ideologica, ben esplicitati dalle traiettorie biografiche di alcuni combattenti. Ecco perché Data Spanish Civil War non si limita a coprire il periodo 1936-1939 di queste traiettorie, ma le considera nel loro complesso. Un'indagine che fa emergere un quadro di opposizione al totalitarismo profondo in termini di tempo (si inizia negli anni '20) e ampio in termini di spazio (per certi versi una vera e propria resistenza europea).

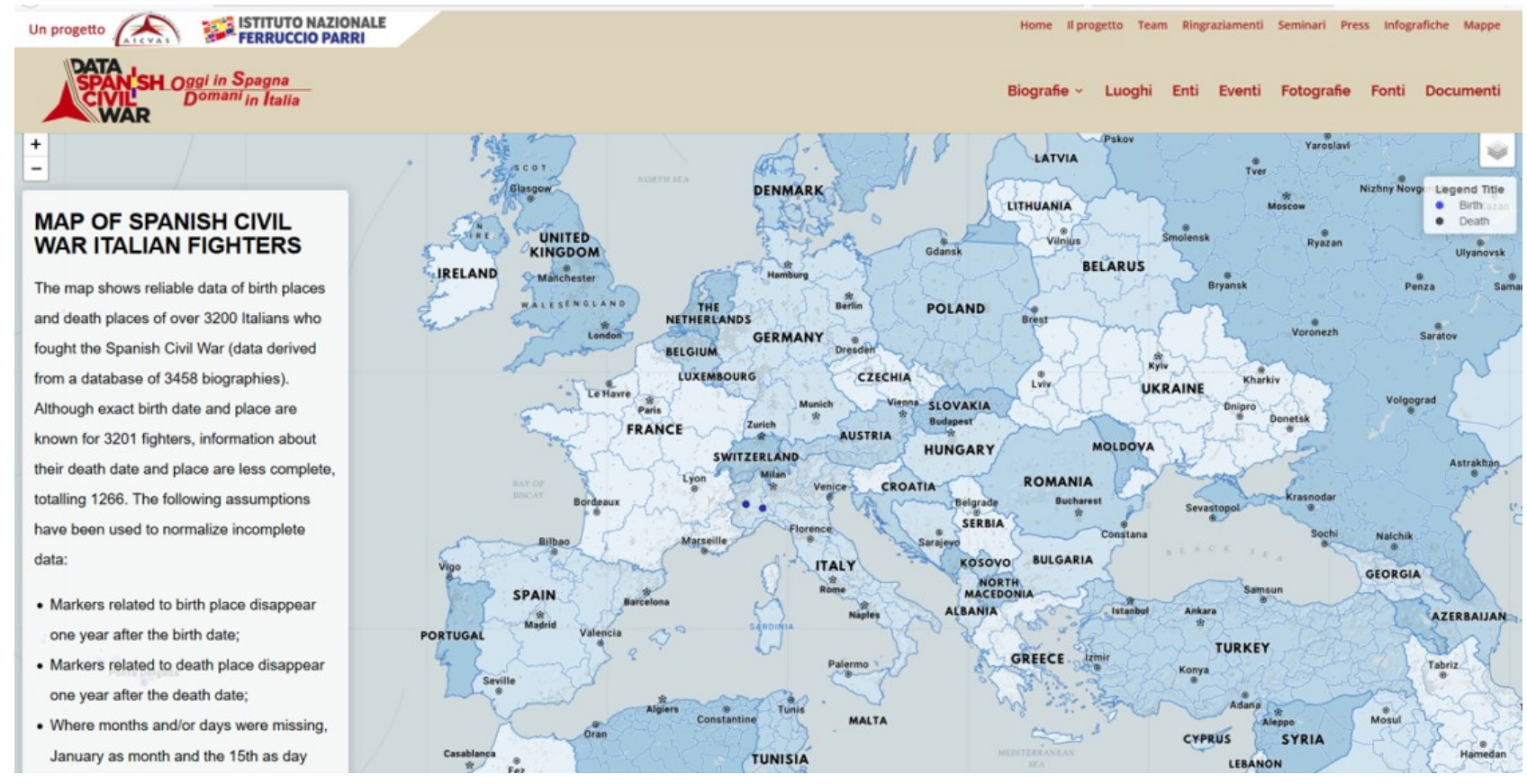

Una doppia anima (ma solo una è public history)

Data Spanish Civil War tuttavia non restituisce solo i profili biografici dei protagonisti: per ognuno di loro infatti è stata effettuata una mappatura delle risorse archivistiche, bibliografiche e digitali in cui sono conservate informazioni o documenti sul loro conto. Di fatto, è come se vi fossero due database distinti, due "anime", collegate tra loro dai nomi e i cognomi dei combattenti.

Naturalmente solo una di queste due "anime" è in grado di coinvolgere il pubblico, di interessarlo e di spingerlo a contribuire alla ricerca e alla produzione del contenuto.

\section{Ogni biografia è una narrazione}

Il riferimento è ovviamente alla parte biografica che è anche uno degli elementi chiave anche per ciò che concerne Ultime lettere.

Per sua stessa natura, infatti, il percorso biografico si presta alla costruzione di narrazioni avvincenti che possono essere restituite in forma di racconto o messa in scena teatrale[22]. Incentrare iniziative o laboratori sulle storie personali o famigliari di alcuni protagonisti (le cui vite sembrano spesso uscire da un romanzo d'avventura) si è dimostrato molto efficace anche da un punto di vista 
didattico. D'altra parte i meccanismi di coinvolgimento del pubblico non sono poi così dissimili, sia dentro che fuori le classi.

\section{Memorie di famiglia}

Quando il pubblico partecipa attivamente? Quando si sente coinvolto al punto da diventare attore e produttore del contenuto storico e non soltanto fruitore?[23] Nel caso delle banche dati prese in esame finora si può dire che questo accade quando intervengono le memorie famigliari o, in minima parte, le piccole ricerche o passioni personali. Non a caso sia per Ultime lettere che per Data Spanish Civil War sono state frequenti le sollecitazioni per emendare informazioni anagrafiche scorrette o per integrare profili biografici lacunosi. Di più: nel caso dei documenti scritti dai condannati a morte e dai deportati della Resistenza, in molti casi sono stati gli stessi eredi a contattare l'Istituto nazionale per fornire le scansioni dei documenti originali autografi. In modo simile nel caso della banca dati sugli antifascisti della guerra di Spagna il contributo dei famigliari è stato importante nell'identificare gli uomini e le donne ritratti in fotografie[24] che erano rimaste senza identità.

Sono sollecitazioni che mancano completamente nel database della Stampa clandestina e che non riguardano la parte delle fonti della banca dati AICVAS e che sono materia naturalmente più indirizzata a una platea esperta e consapevole, o che ha necessità di avvalersi di quei repertori per scopi di studio o ricerca.

\section{Banche dati e territorio: l'Atlante delle stragi e Pietre nella rete}

Un'ultima tipologia di database si lega a dati che rappresentano il territorio, come nell' Atlante delle stragi naziste e fasciste e nelle Pietre nella rete. Due prodotti, del resto, agli antipodi sotto molti punti di vista.

L'Atlante nasce da un progetto di ricerca dell'Istituto nazionale Ferruccio Parri, finanziato dal Fondo italo-tedesco per il futuro[25]. È un database dal respiro nazionale che ha lo scopo censire tutti gli episodi di violenza mortale perpetrati in Italia dai nazisti e dai fascisti dall'armistizio del 1943 alla fine della Seconda guerra mondiale nel 1945.

Pietre nella rete è invece un progetto dell'Associazione PopHistory finanziato dalla Regione Emilia-Romagna. La banca dati ha un focus sulla Prima guerra mondiale e su come essa ha impattato sulla storia e la memoria di alcuni comuni emiliano-romagnoli.

I presupposti dei due strumenti sono assai differenti. Da un lato c'è un progetto di ricerca classico con una lunga gestazione (le prime ricerche sono della seconda metà degli anni Novanta, mentre la banca dati è on line dal 2016) e che solo nella sua ultima fase ha visto sciogliere la riserva se affidarsi a un esito cartaceo o digitale. Dall'altro un prodotto dichiaratamente di public history che mira a restituire la memoria dei caduti della Grande guerra e la storia dei monumenti fisici dei singoli comuni, degradati dal tempo o cancellati dalle politiche di governo del territorio[26]. 


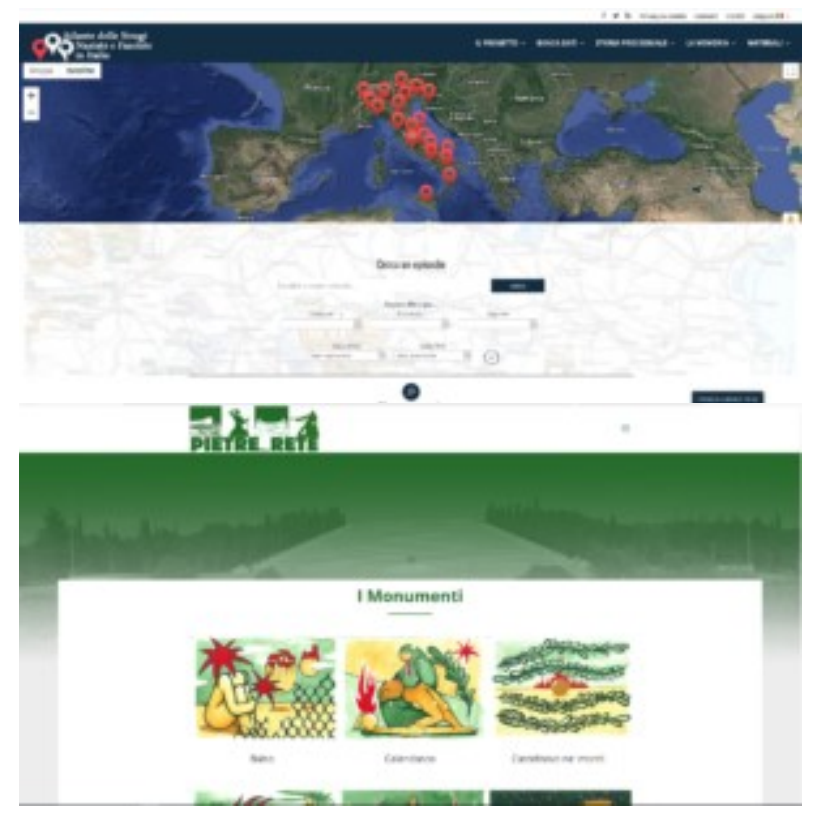

\section{Comunità e memorie locali}

Entrambi i casi tuttavia sono simili per la focalizzazione sulle memorie delle comunità locali e del territorio. In particolare l'Atlante, che fa riferimento ad avvenimenti più recenti e per certi versi più laceranti del tessuto sociale, ha fatto scaturire una generosa contribuzione alla produzione dei contenuti storici da parte di ricercatori locali, associazioni o famigliari (avvenuta prevalentemente nella fase che ha seguito la sua messa on line). L'intera operazione, comunque, è stata monitorata ed esaminata dalla segreteria di ricerca, che si è occupata di selezionare, controllare, accogliere o rigettare le proposte di modifica o integrazione.

In Pietre nella rete questa operazione era parte dalla raccolta della documentazione fin dalle fasi iniziali: nella costruzione di ogni archivio dei caduti e di ogni testo descrittivo presente nel sito ci si è avvalsi della collaborazione delle varie istituzioni locali e, in qualche caso, di privati cittadini. $\mathrm{E}$ tuttavia questa partecipazione ha avuto esiti alterni. Ragionando sul public engagement è curioso osservare come abbia funzionato meglio uno strumento non pensato per essere di public history, rispetto a un altro progettato e realizzato per essere tale.

Nonostante ciò, Pietre nella rete ha diversi altri elementi di valore "public": in particolare una rara mescolanza di linguaggi al servizio della ricerca e della produzione storica. Al suo interno compaiono canzoni d'epoca (riprodotte dal Centro studi musica e Grande guerra) brevi narrazioni biografiche di tipo letterario per ricostruire le biografie di alcuni protagonisti, più noti o semisconosciuti.

Resta il dato centrale che l'approfondimento per biografie si conferma come uno strumento utilissimo per incentivare il coinvolgimento.

\section{Tirando le somme}

Digital history, public history e digital/public memory sono, dunque, sfere distinte ma con confini tra loro tutt'altro che netti. Le aree di contatto e contaminazione sono molteplici. È però evidente 
che il digitale in sé non basta a identificare un prodotto di public history, almeno intesa in senso "forte" e non come semplice comunicazione o divulgazione storica.

Ci si potrebbe chiedere provocatoriamente se una conferenza storica di tipo accademico fatta a teatro sia etichettabile come public history. Senza voler peccare di dogmatismo, la risposta non potrà che essere negativa, perché non è il teatro, cioè il luogo, a caratterizzare la public history. Per lo stesso motivo una banca dati archivistica o un repertorio di fonti (com'è ad esempio www.stampaclandestina.it) non rappresentano un prodotto di public history per il semplice fatto di essere in forma digitale. Possono diventarlo se trattano argomenti in grado di suscitare interesse nel grande pubblico o hanno in nuce una predisposizione particolare alla partecipazione collettiva (com'è accaduto per www.straginazifasciste.it), quand'anche non con una progettualità già ben chiara in partenza (ed è il caso di www.pietrenellarete.it).

Certamente la capacità del digitale di mescolare facilmente i linguaggi e i registri, di usare la multimedialità, di favorire uno scambio più capillare delle informazioni, può creare i presupposti per avviare $\mathrm{o}$ coadiuvare pratiche di public history, sfruttando argomenti già di per loro noti al pubblico o particolari forme di narrazione della storia (è il caso delle biografie e di progetti come www.ultimelettere.it).

Come sottolineato in introduzione, occorre tuttavia tenere sempre a mente che il pubblico raggiungibile on line è solo potenziale (public access). Soprattutto nella società iperconnessa e ipercomunicativa odierna, in cui veniamo investiti ogni giorno da un sovraccarico di informazioni e di nozioni, il pubblico (ma anche gli studenti in classe) ha bisogno di essere sollecitato, convinto e stimolato alla partecipazione, per giungere a quel vero e proprio coinvolgimento (public engagement) che contraddistingue la public history.

\section{Bibliografia}

- M. Carrattieri, Per una public history italiana, in "Italia contemporanea", n. 289, aprile 2019

- T. Cauvin, Public history, a textbook of practice, Taylor \& Francis Ltd, 2016

- M. Flores, S. Pivato, A proposito di Public History, in "Novecento.org", n. 13, febbraio 2020. DOI: [12977/nov208] url consultata il 19 ottobre 2019

- R. Minuti, Il web e gli studi storici. Guida critica all'uso della rete, Carocci, Roma 2015

- Stampa clandestina : 1943-1945 : storie, fonti, strumenti per la didattica, INSMLI, Milano 2017 [http://www.stampaclandestina.it/wp-content/uploads/2017/01/Stampa-clandestinaQuaderno-didattico.pdf], url consultata il 19 ottobre 2019

\section{Sitografia}

- Atlante delle stragi naziste e fasciste in Italia [straginazifasciste.it]

- Data Spanish Civil War [antifascistispagna.it]

- Pietre nella rete [pietrenellarete.it]

- Stampa clandestina 1943-45 [stampaclandestina.it]

- Ultime lettere di condannati a morte e di deportati della Resistenza [ultimelettere.it] 
Note:

[1] R. Biles, The human tradition in urban America, Rowman \& Littlefield Publishers, 2002, p. 128.

[https://books.google.pl/books?id=DqayY98DDDcC\&pg=PA128\&lpg=PA128\&dq=church+garage + automobile\&source $=$ bl\&ots $=$ NMnjn43sFY\&sig $=\mathrm{I}-$

YoAzHs5ChdCEE61sOEK073WQY\&hl=pl\&sa=X\&ei=sUsCT6-

QE8vJsgb4qfmDAg\#v=onepage\&q= church\%20garage\%20automobile\&f=false] url consultata il 19 ottobre 2019

[2] M. Carrattieri, Per una public history italiana, in "Italia contemporanea", n. 289, aprile 2019, p. 112

[3] T. Cauvin, Public history, a textbook of practice, Taylor \& Francis Ltd, 2016, p. 174

[4] P. Svensson, The Landscape of Digital Humanities, in Digital Humanities, 2010

[5] R. Minuti, Il web e gli studi storici. Guida critica all'uso della rete, Carocci, Roma 2015, p. 11

[6] D. Cohen, Interchange: The Promise of Digital History, in Journal of American History, Volume 95, Issue 2, September 2008, Pages 452-491, https://doi.org/10.2307/25095630

[7] Carrattieri, 2019, p. 113

[8] Cauvin, 2016, p. 177

[9] Minuti, 2015, p. 11

[10] Cauvin, 2016, p. 177

[11] Cauvin, 2016, p. 178

[12] Carrattieri, 2019, p. 113

[13] D. Bidussa, Maturità 2019, impariamo a insegnare la storia, in "Il Sole 24ore", 28 febbraio 2019, [https://www.ilsole24ore.com/art/impariamo-insegnare-storia-ABSvF6YB], url consultata il 15 luglio 2019

[14] M. Flores, S. Pivato, A proposito di Public History, in "Novecento.org", n. 13, febbraio 2020. DOI: [10.12977/nov208], url consultata il 19 ottobre 2019

[15] S. Noiret, Introduzione, in P. Bertella Farneti, L. Bertuccelli, A. Botti, Public History. Discussioni e pratiche, Mimesis, Milano-Udine 2017, p. 18.

[16] Sulla questione della memoria e della storia si rimanda al libro di David Bidussa, Dopo l'ultimo testimone, Einaudi, Torino 2009, in particolare il capitolo 6, Fare i conti con il passato, pp. 79-114..

[17] Si veda ad esempio il caso di Leone Ginzburg, catturato nella stamperia clandestina di Italia libera, a Roma (http://www.ultimelettere.it/?page $\mathrm{id}=35 \&$ ricerca $=250$, url consultata il 19 ottobre 2019). 
[18] Alessandro Portelli, nell'introduzione di L'ordine è già stato eseguito, lo scriveva per le fonti orali, ma il concetto è applicabile anche in questo caso.

[19] G. Perona, Presentazione, in "Ultime lettere di condannati a morte e di deportati della Resistenza italiana", [http://www.ultimelettere.it/?page id=53], url consultata il 19 ottobre 2019.

[20] Ancora Perona in "Ultime lettere di condannati a morte e di deportati della Resistenza italiana", [http://www.ultimelettere.it/?page id=53], url consultata il 19 ottobre 2019.

[21] Per approfondire si consiglia di leggere l'articolo di Enrico Acciai Memorie difficili.

Antifascismo italiano, volontariato internazionale e guerra civile spagnola, pubblicata nel n. 7/2011 della rivista "Diacronie".

[22] A Milano l'Istituto nazionale Ferruccio Parri ha collaborato a un progetto con il Teatro della cooperativa, che ha utilizzato la banca dati "Ultime lettere" e coinvolto diversi studenti e studentesse degli istituti superiori del quartiere Niguarda, in cui il teatro ha sede.

[23] È il concetto, molto trasversale, del prosumer, parole inglese che è sintesi di producer e consumer; si riferisce al destinatario di beni e di servizi che non si limita al ruolo passivo di consumatore, ma partecipa attivamente alle diverse fasi del processo produttivo.

[24] [http://www.antifascistispagna.it/?page_id=2], url consultata il 18 ottobre 2019.

[25] [http://www.straginazifasciste.it/?page id=9], url consultata il 18 ottobre 2019.

[26] Esemplare il caso di Vetto (RE), dove il vecchio monumento con i nomi dei caduti è stato sostituito da una stele in cui non v'è più traccia dell'identità di chi ha perso la vita nel conflitto [http://www.pietrenellarete.it/vetto/], url consultata il 19 ottobre 2019 